\title{
Adaptive sampling with Bayesian compressive sensing in radar sensor networks and image
}

\author{
Wei Wang and Baoju Zhang*
}

\begin{abstract}
The theory of Bayesian compressive sensing is briefly introduced and the differential entropy for recovery signal is deduced. An evaluation index based on differential entropy is devised and the adaptive compressive sampling procedure without any prior information of the measured signals is presented in block manner. Numerical simulations on random step signal and real radar signal and 2D image verify that the proposed adaptive sampling algorithm has good performance. This novel algorithm offers great potential for adaptive compressive sampling in real-time radar signal and image.
\end{abstract}

Keywords: Adaptive sampling, Sparse Bayesian learning, Compressive sensing, Real-time signal sampling

\section{Introduction}

The emerging theory of compressive sensing (CS) has provided a new framework for signal acquisition and it has gained increased interests over the past few years. CS is an alternative to the Nyquist rate for the acquisition of 'sparse' signals. Consider a signal that is sparse in some basis (often using a wavelet-based transform coding scheme). The basic idea of CS is projecting the highdimensional signal onto a measurement matrix, which is incoherent with the sparsifying basis, resulting to a lowdimensional sensed sequence. Then with a relatively small number of appropriately designed projection measurements, the underlying signal may be recovered exactly $[1,2]$. In contrast to the common framework of first collecting as much data as possible and then discarding the redundant data by digital compression techniques, CS seeks to minimize the collection of redundant data in the acquisition step.

Despite the tremendous reduction in the number of observations required for provable success of CS methods, the traditional CS methods do suffer several drawbacks [3]. Typically, existing CS architectures are not adaptive and the number of samplings is determined before the acquisition process begins with no feedback during the acquisition process on the improved quality [4]. Even more, in measuring signal, such as radar signal, where its interest is

\footnotetext{
* Correspondence: wdxyzbj@163.com

College of Physical and Electronic Information, Tianjin Normal University, Tianjin, China
}

time-varying, there may not be sufficient prior knowledge to decide appropriate CS observations.

To improve the limitations of traditional CS, several recent studies through the use of adaptive acquisition of CS observations have been proposed. A simple data-adaptive procedure based on iterative refocusing scheme was described in [3]. Although it did not require explicit knowledge of the observation noise power and enjoy the benefit of lower implementation complexity, it did not provided proper index for determining the numbers of CS observations. An algorithm called adaptive compressed sampling has been presented in [4]. While exploit wavelet tree structures found in natural images to replace the 'universal' acquisition of incoherent measurements, the approach based on wavelet transform limit its application. The sparsity adaptive matching pursuit (SAMP) was presented in [5]. It is a novel iterative greedy algorithm and can reconstruct signal without prior information of the signal sparsity. But, the experimental results confirmed that the SAMP was appropriate for reconstructing compressible sparse signal where its magnitudes were decayed rapidly. A Bayesian formalism for estimating the underlying signal based on CS measurements, called Bayesian Compressive Sensing (BCS), was employed in [6,7]. It estimated "error bars" for underlying signal to give a measure of confidence in the inverted signal, but it did not move forward to propose adaptive sampling strategy.

Here, we further elaborate the BCS and present an index to determine how many samples are enough for

\section{然 Springer}



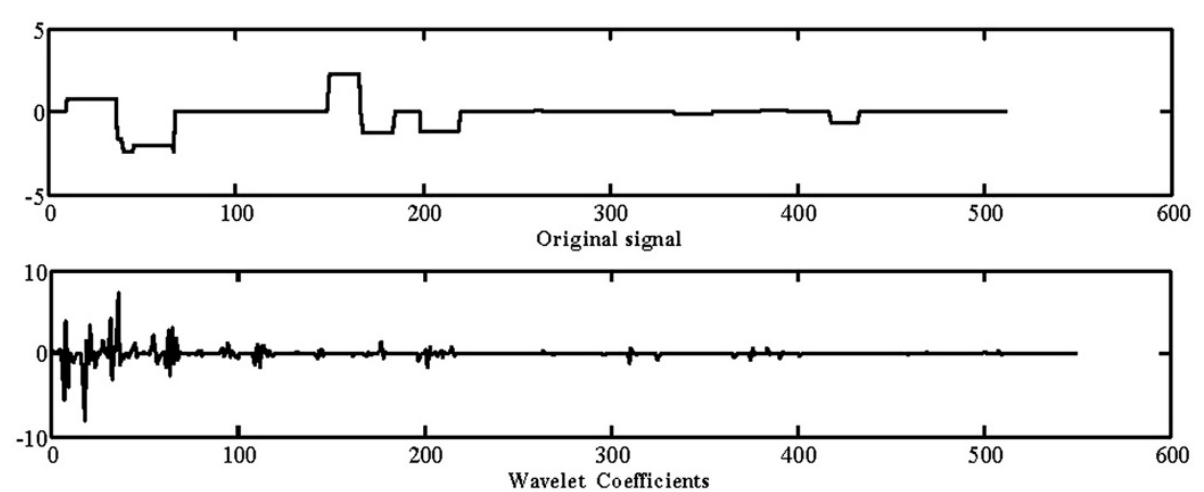

Figure 1 The original signal and its wavelet coefficients.

faithful CS reconstruction without prior knowledge of underlying signal. The remainder of the article is organized as follows. In the following section, the basics of BCS as needed for this article are summarized. In Section "Adaptive compressive measurement procedure", we proposed our adaptive procedure and evaluation index. Example results are presented in Section "Experimental demonstrate". Conclusion and future work are discussed in Section "Conclusion".

\section{Background of BCS}

$\mathrm{CS}$ has received considerable attention recently. The theory of CS states that a signal may be captured with a small set of non-adaptive, linear measurements as long as the signal is sparse in some basis (such as DCT, wavelet) $[8,9]$. We can write in matrix notation:

$$
y=\Phi x+n=\Phi \Psi \theta+n=\Theta \theta+n
$$

where $\Phi$ is a $M \times N$ measurement matrix, $y \in R^{M}$ is the vector of samples observed, and $n$ is an $M \times 1$ vector that represents measurement noise and error. $\theta$ is the transform coefficient vector of signal $x$ under orthonormal basis $\Psi$, i.e., $\theta=\Psi^{T}=x$. When $\theta$ contains $K \ll N$ non-zero elements, we need to acquire only $M=$ $O(K \log (N / K))$ random measurements to recover the signal $x$.
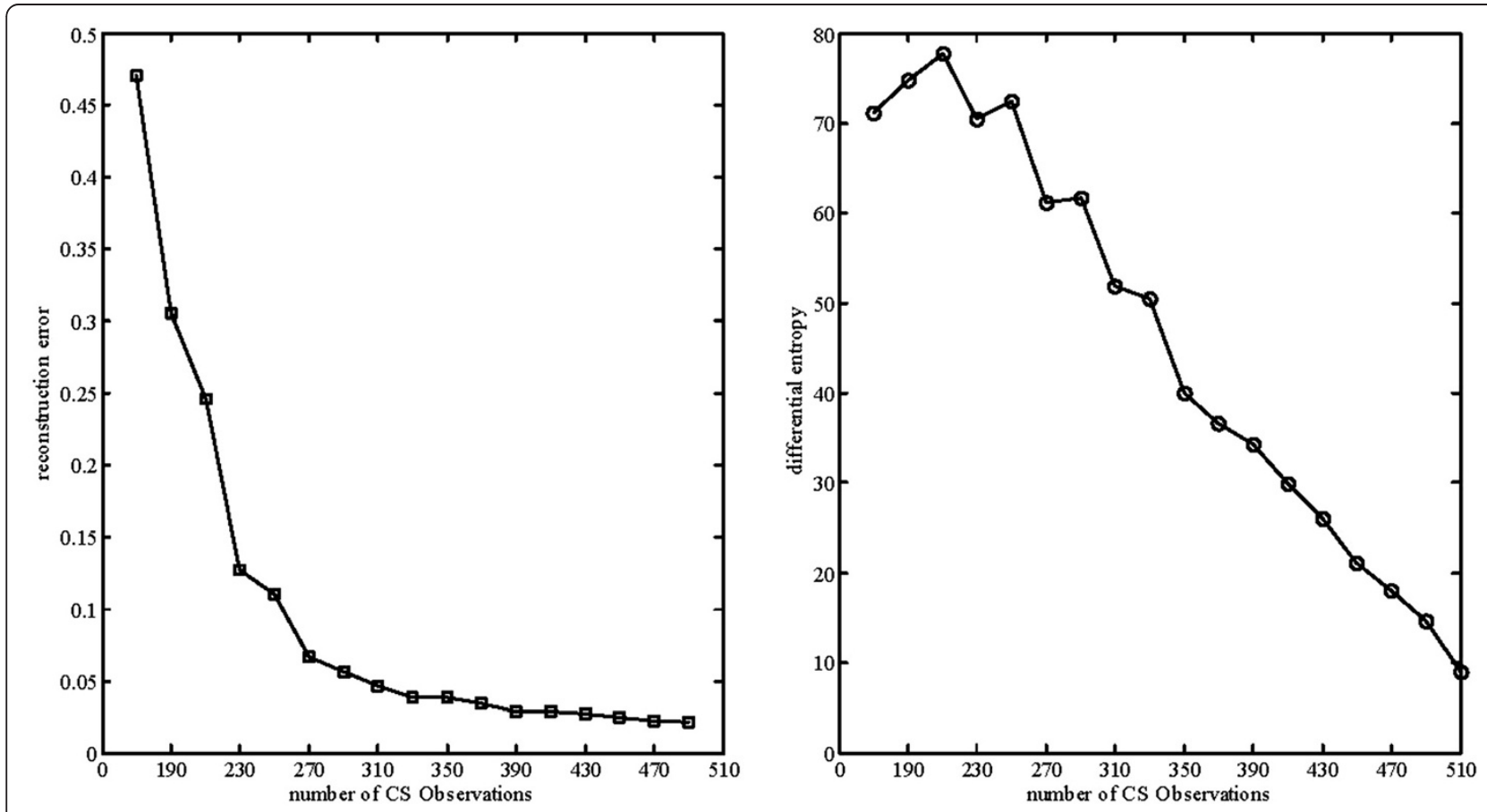

Figure 2 The chart of varying trend of reconstruction error and differential entropy with $p=150, q=20$. 

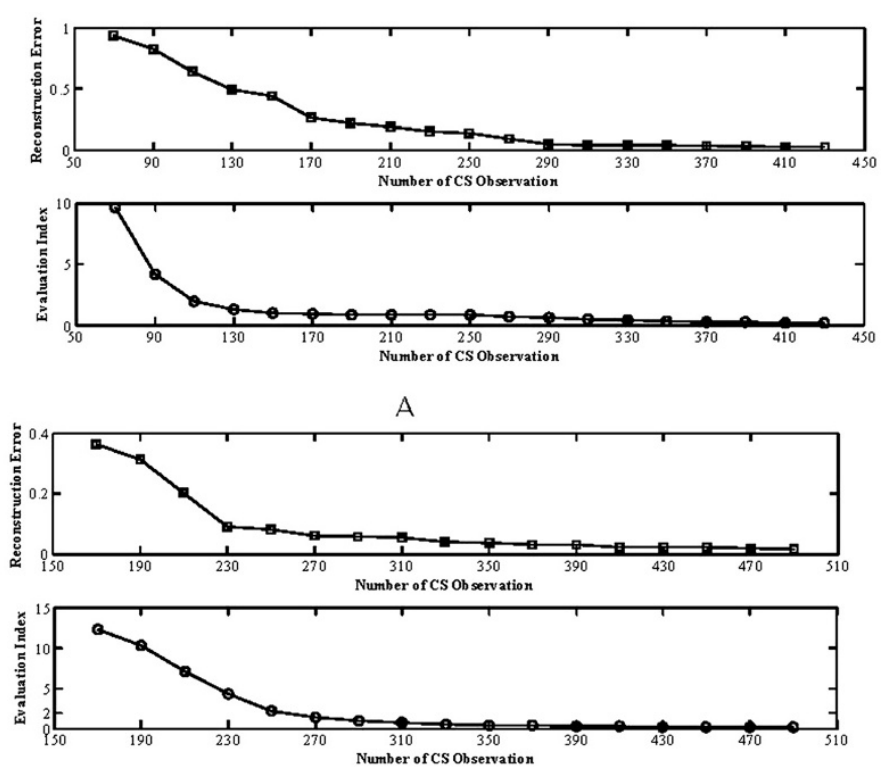

$\mathrm{B}$
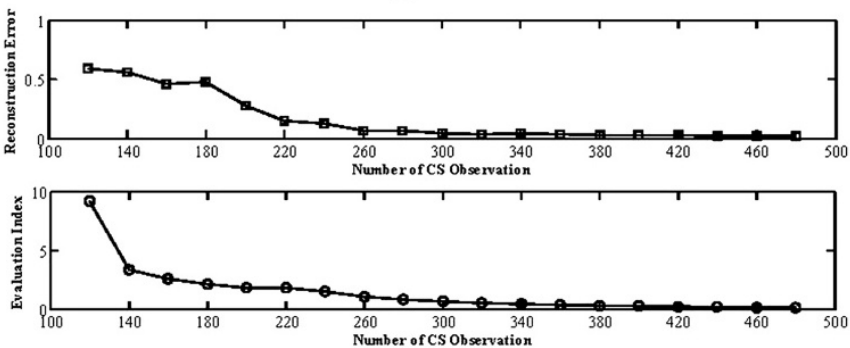

C
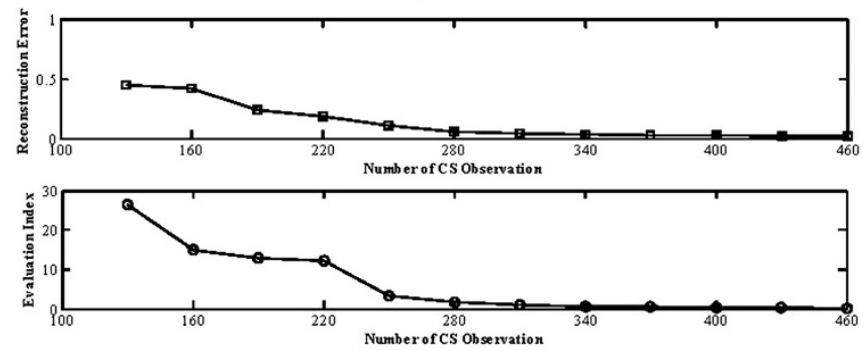

$\mathrm{D}$
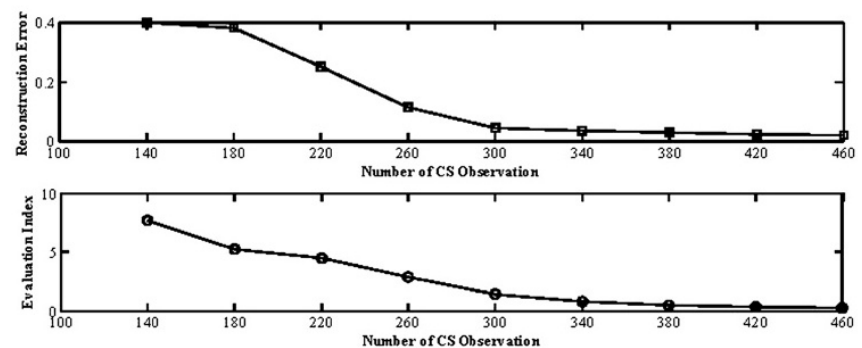

E

Figure 3 The chart of varying trend of reconstruction error and evaluation index with different $\boldsymbol{p}, \boldsymbol{q}$. (A) $p=50, q=20 .(\mathbf{B}) p=150, q=20$. (C) $p=100, q=20$. (D) $p=100, q=30$. (E) $p=100, q=40$. 
To recovery the signal $x$ from CS observations, many algorithms have been presented, such as basis pursuit algorithm, matching pursuit (MP), compressed sampling MP , iteratively hard thresholding algorithm, and so on. But, these algorithms have no feedback parameters to evaluate the acquisition process and cannot adaptively observe the time-varying radar signal. The BCS consider the inversion of compressive measurement from a Bayesian perspective, which can yield "error bars" on the estimated the signal $x$ and can be used to guide the optimal design of adaptive CS measurements [6]. In BCS, each element of transform coefficients $\theta$ is defined a zero-mean Gaussian prior:

$$
p(\theta \mid \alpha)=\prod_{i=1}^{M} N\left(\theta_{i} \mid 0, \alpha_{i}^{-1}\right)
$$

with $\alpha_{i}$ the precision of a Gaussian density function and $\alpha$ the hyper parameter considered as Gamma prior:

$$
p(\alpha \mid a, b)=\prod_{i=1}^{N} \Gamma\left(\alpha_{i} \mid a, b\right)
$$

where $a$ and $b$ are the shape and scale parameters of gamma distribution, respectively. So, the overall prior on $\theta$ can be evaluated as

$$
p(\theta \mid a, b)=\prod_{i=1}^{N} \int_{0}^{\infty} N\left(\theta_{i} \mid 0, \alpha_{i}^{-1}\right) \Gamma\left(\alpha_{i} \mid a, b\right) d \alpha_{i}
$$

where $\theta_{i}$ plays the role of observed data and $N\left(\theta_{i} \mid 0, \alpha_{i}^{-1}\right)$ is a likelihood function. Given the CS observation $y$ and the random measurement matrix $\Phi$, orthonormal basis $\Psi$, the posterior for $\theta$ can be solved by the relevance vector machine with mean and covariance:

$$
\begin{aligned}
& \mu=\alpha_{0} \Sigma \Theta^{T} y \\
& \Sigma=\left(\alpha_{0} \Theta^{T} \Theta+A\right)^{-1}
\end{aligned}
$$

where $A=\operatorname{diag}\left(\alpha_{1}, \alpha_{2}, \cdots \alpha_{M}\right)$ and $\alpha_{0}$ is the reciprocal of noise variance when the noise $n$ represented by a zero-mean Gaussian distribution. Further, the estimated posterior on the signal $x$ is a multivariate Gaussian distribution: $E(x)=\Theta \mu, \operatorname{Cov}(x)=\Theta \Sigma \Theta^{T}$. So, the differential entropy for $x$ satisfies:

$$
h(x)=-\int p(x) \log p(x) d x=1 / 2 \log |\Sigma|+\text { const }
$$

where const is independent of $\Theta$. The differential entropy $h(x)$ provides a measure of uncertainty $x$, i.e., it gives a way to determine how many measurements are enough for faithful CS reconstruction. In this article, we will introduce an adaptive CS measurement procedure base on the $h(x)$ for radar signal and 2D image.

\section{Adaptive compressive measurement procedure}

In the theory of $\mathrm{CS}$, the number of CS observations should be more than $C(K \log (N / K))$ for the high precision signal recovery. But, it would be much larger in reality, due to the unknown of $K$-the sparsity of underlying signal-in advanced. It enormously limits the application of CS in some other real-time signals.

In this article, an adaptive compressive sampling procedure based on BCS and differential entropy $h(x)$ has been proposed. The algorithm is as follows:

1. Construct measurement matrix $\Phi \in R^{N \times N}$ based on Equation (1);
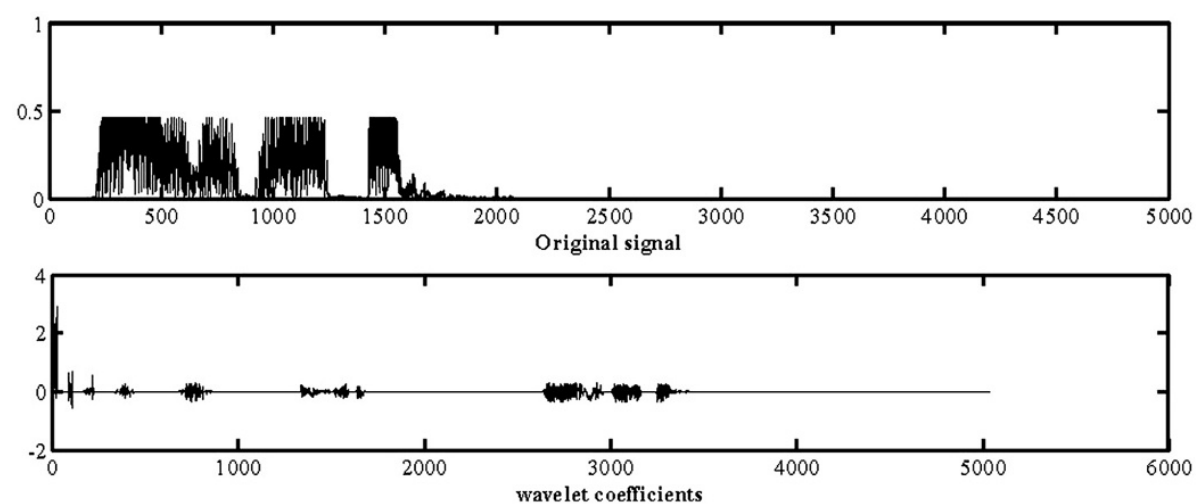

Figure 4 The radar signal and its wavelet coefficients. 

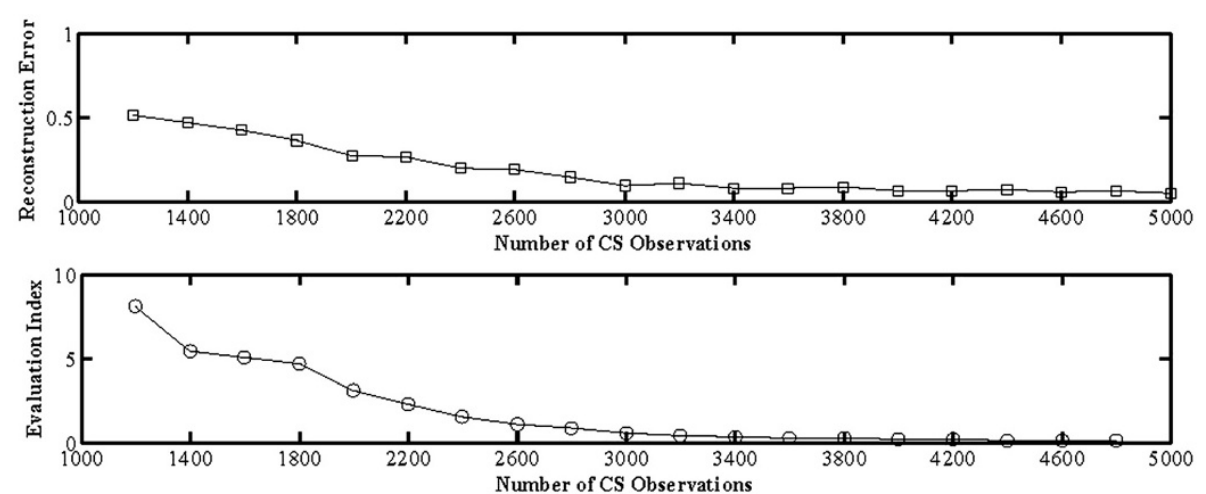

Figure 5 The chart of reconstruction error and evaluation index with $p=1000, q=200$.

2. Use the first $p$ rows of $\Phi$ to obtain CS measurements $\mathrm{y} \in R^{P}$, and solve the $h_{1}(x)$ based on Equation (7);

3. Use the next $q$ rows of $\Phi$ to obtain CS measurements $y \in R^{\mathrm{q}}$, and solve the $h_{2}(x)$ based on Equation (7);

4. Established the adaptive evaluation index $S_{i d}(r)=$ $\frac{\bar{h}(x)}{\sum_{i=1}^{r}\left|h_{i}(x)-\bar{h}(x)\right|}$, where $\bar{h}(x)=\frac{\sum_{i=1}^{r} h_{i}(x)}{r}, r \geq 2$

5. Compare $S_{i d}(r)$ with $\in$ which is the end tag. If $S_{i d}(r) \leq \epsilon$, put through the measurement and recover the signal $x$ from CS observation. If not, go back to step () and continually use the next new $q$ rows of $\Phi$ to obtain CS measurements $\mathrm{y} \in R^{q}$, and solve the $h_{i}$ $(x)$, then go back to step 4 .

In this algorithm, the signal acquisition is conducted in a block manner with the block size $q$. The $p$ is the first block size of CS observations and a proper $p$ can reduce the measuring time. The adaptive evaluation index $S_{i d}(r)$ deduced by the statistical feature of differential entropy $h(x)$ is used to judge the uncertainty of the recovered signal. The $\epsilon$ is a small positive number for procedure end tag and the measuring procedure would stop when evaluation index $S_{i d}(r)$ is less than $\in$. That is, it can achieve high reconstruction accuracy at this moment. Due to no prior knowledge of original signal used, this algorithm is adaptive in CS observations. Meanwhile, the $S_{i d}(r)$ can be solved with the next block CS observation acquired simultaneously, so it is real time and extremely applicable for radar signal measurement.

\section{Experimental demonstrate}

To illustrate the effectiveness of the proposed adaptive algorithm, we test its performances on random signal, actual radar signal and 2D image. In the experiments, we chose random Gaussian matrix for measurement matrix $\Phi$, and ' $d b 4$ ' wavelet for orthonormal basis $\Psi$. We used the BCS package available online at http://people.ee. duke.edu/ lcarin/BCS.html, which we modified so that it would match the adaptive compressive measurement procedure. All the computations were run on a $3.20 \mathrm{GHz}$ Inter Core i5 desktop PC with 2 GB RAM.

\section{Random signals simulations}

In Figure 1, we generated a step signal with random step position, step width, step amplitude, and the length
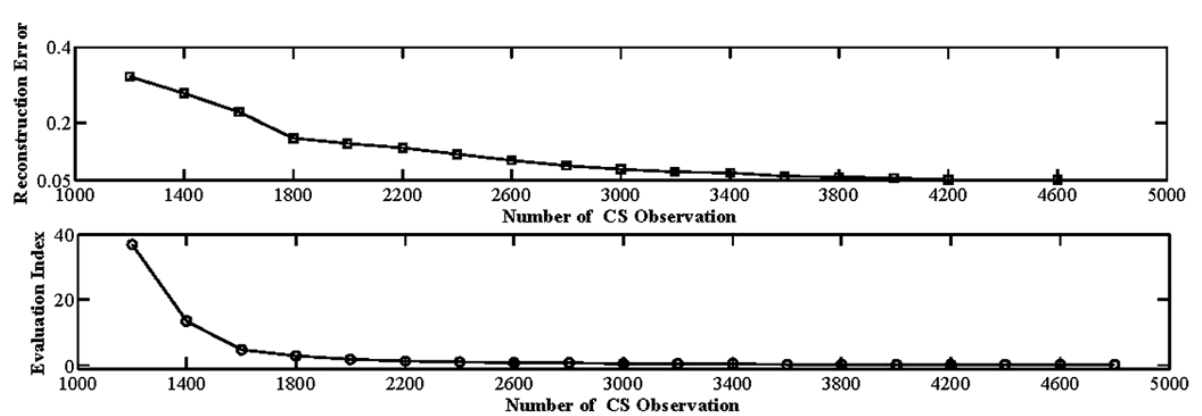

Figure 6 The chart of reconstruction error and evaluation index with $p=1000, q=200$. 

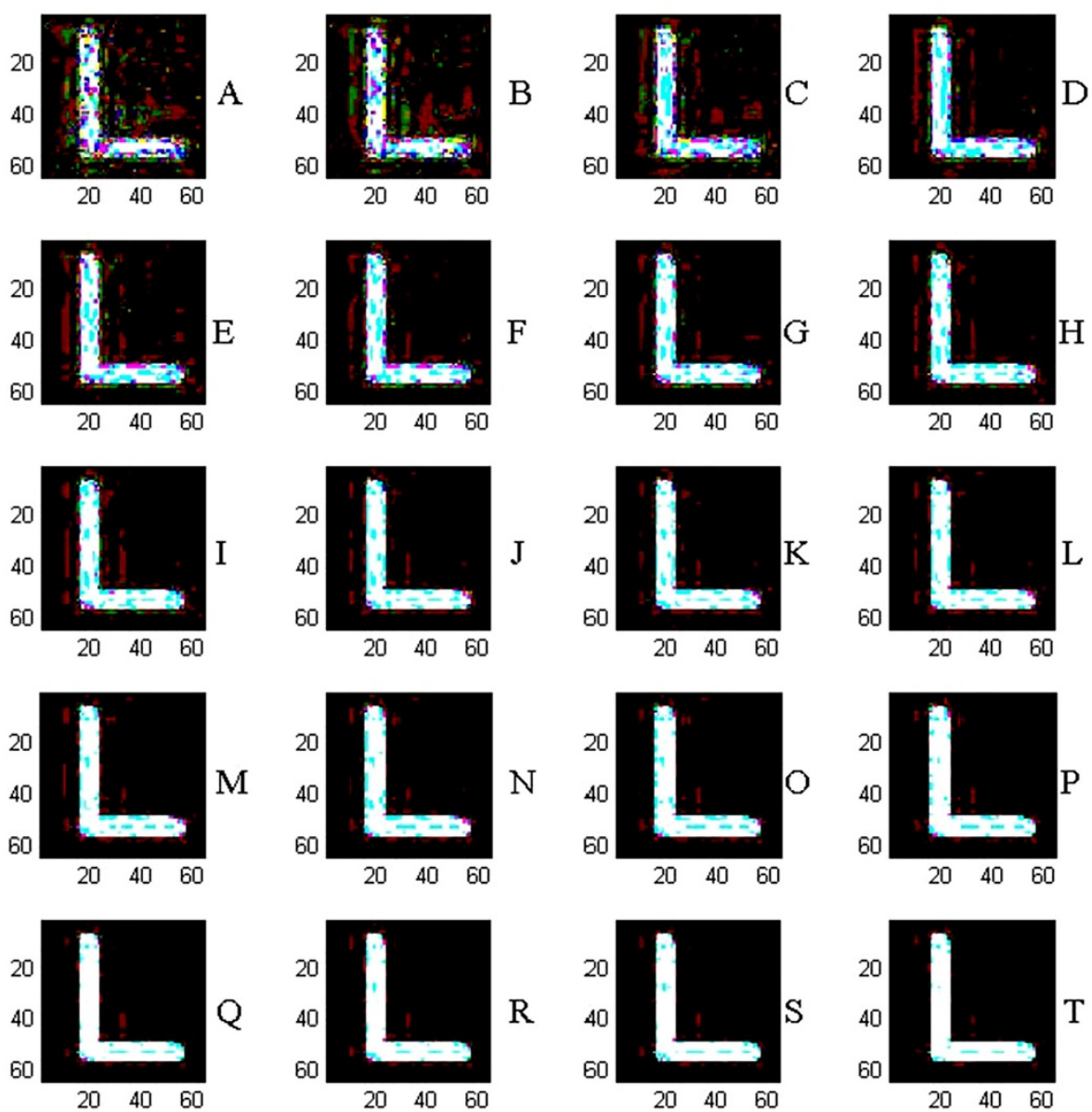

Figure 7 The recovery images with increasing samplings.

$N=512$. The waveform of random step signal and its wavelet transform coefficients are shown in Figure 1. It is clear that the coefficients are sparse and the signal can be measured with CS compressively.

First, in the simulations, we tested whether the differential entropy $h(x)$ can be used to determine how many samplings are enough for faithful CS reconstruction directly or not. The varying trend of reconstruction error $R_{\text {err }}=\frac{\left|\left(x-x^{\prime}\right)\right|_{2}}{\|x\|_{2}}$ and the differential entropy $h(x)$ were showed in Figure 2 when $p=150 ; q=20$, where $x^{\prime}$ is the recovery signal.

It is clearly showed that the differential entropy $h(x)$ is fluctuant because of Bayesian estimation theory, and it has the different varying trend with reconstruction error. That is, $h(x)$ cannot be used as the adaptive evaluation index directly.

Secondly, we study the performance of different $p$ and $q$ and depict the varying trend of reconstruction error $R_{e r r}=\frac{\left|\left(x-x^{\prime}\right)\right|_{2}}{\|x\|_{2}}$ and evaluation index $S_{i d}(r)$.

Figure 3A-E demonstrates the varying curve of reconstruction error $R_{\text {err }}$ and evaluation index $S_{i d}(r)$ with $p=50,100,150 ; q=20 \quad$ and $q=20,30,40 ; p=100$, respectively. From the simulation results, it suggests that: (1) the curve of $R_{\text {err }}$ and $S_{i d}(r)$ has similar change trend. In other words, the evaluation index $S_{i d}(r)$ can adaptively reflect the reconstruction error with CS observations increasing, i.e., it can be used for stop tag of CS measurement; (2) from Figure $3 \mathrm{~A}-\mathrm{C}$ and B,D,E, respectively, we can see that there are good adaptive evaluation results with fewer $p$ and $q$, i.e., with larger number of block CS observations. In other words, we can achieve better compression ratio with less number of CS observation through favorable $p$ and $q$. But, in real-time measuring, the time consume should be considered to select favorable $p$ and $q$.

\section{Real radar signals experiment}

Radar signal systems are in high demand in many civilian, military, and biomedical applications. The states of art radar systems apply a large bandwidth and an increasing number of channel produce huge amount of data $[10,11]$. And often the data handling is the most crucial matter of design. Achieving adequate wideband radar signal (which can be compressed into a short 
duration pulse) requires both a high sampling frequency and a large dynamic range. Currently available A/D conversion technology is a limiting factor in the design of ultra wideband radar systems, because in many cases the required performance is either beyond what is technologically possible or too expensive [12]. Moreover, radar sensor networks (RSN) have recently been considered to overcome the performance degradation of a single radar. Due to the expansion of data introduced to RSN, the compression of received data is a design challenge of future RSN. A topic addressed by the emerging field of CS is a radically new approach to solve these challenges.

In this part, we use the adaptive CS algorithm to measure the real radar signal. The radar signal that we use the amplitude part and ten times amplified is shown in Figure 4. The figure also shows that only small amount of wavelet coefficients are non-zero, i.e., the radar signal is sparse and can be observed by CS. Figure 5 shows that the evaluation index can reflect the change trend of reconstruction error well with no prior knowledge about radar signal.

\section{D image experiment}

In the following experiment, the adaptive CS algorithm was used to measure the $2 \mathrm{D}$ image which was white " $L$ " in black background and it has 64\%64 pixels. From Figure 6, it can be showed obviously that the evaluation index has the similar varying trend with the reconstruction error. So, the presented adaptive sampling algorithm could be used in 2D signal measurement too. The recovery images of each sampling step are displayed in Figure 6 and we can see that the recovery images are nearly the same with the numbers of CS observation increasing, especially in Figure 7 (M-T). It also demonstrates that results given in Figure 6.

\section{Conclusion}

We studied an adaptive CS algorithm based on BCS and devised an evaluation index constructed by differential entropy of estimated signal. The experimental results demonstrated that the procedure consistently effective for adaptive CS measurement.

However, there are some challenges to be overcome before an actual adaptive CS algorithm for real-time radar signal will become a reality. First, the fluctuation of stop tag should be analyzed due to the random observation. Second, a faster recovery algorithm must be designed to handle real-world signal acquisition with sufficient computational efficiency.

\section{Competing interests}

Both authors declare that they have no competing interests.

\section{Acknowledgment}

The authors appreciate the CS research community in Duke University for their BCS code, it made the experiments in this article very convenient. This research was supported by the High Education Science \& Technology Foundation Planning Project of Tianjin (20100716), the Tianjin Natural Science Foundation (10JCYBJC00400), and the Tianjin Younger Natural Science Foundation (12JCQNJC00400).

Received: 18 July 2012 Accepted: 24 July 2012

Published: 16 August 2012

\section{References}

1. Y. Rivenson, A. Stern, Compressed imaging with a separable sensing operator. IEEE Signal Process. Lett. 16(6), 449-452 (2009)

2. L. He, L. Carin, Exploiting structure in wavelet-based bayesian compressive sensing. IEEE Trans. Signal Process. 57(9), 3488-3497 (2009)

3. J. Haupt, R. Nowak, R. Castro, Adaptive sensing for sparse signal recovery, in Digital Signal Processing Workshop and 5 th IEEE Signal Processing Education Workshop (, Marco Island, FL, 2009), pp. 702-707

4. A. Averbuch, D. Shai, D. Shay, Adaptive compressed image sensing using dictionaries. SIAM J. Imag. Sci. 5(1), 57-89 (2012)

5. T.T. Do, G. Lu, N. Nam et al., Sparsity adaptive matching pursuit algorithm for practical compressed sensing, in The 42nd Asilomar Conference on Signals. Systems and Computers, Pacific Grove, CA 10, 581-587 (2008)

6. J. Shihao, X. Ya, L. Carin, Bayesian compressive sensing. IEEE Trans. Signal Process. 56(6), 2346-2356 (2008)

7. S. Ji, D. Dunson, L. Carin, Multitask compressive sensing. IEEE Trans. Signal Process. 57(1), 92-106 (2009)

8. E. Candès, T. Tao, Near-optimal signal recovery from random projections: universal encoding strategies. IEEE Trans. Inf. Theory 52(12), 12 (2006)

9. E. Candès, M. Wakin, An introduction to compressive sampling-a sensing/ sampling paradigm that goes against the common knowledge in data acquisition. IEEE Signal Process. Mag. 25(2), 21-30 (2008)

10. M.A. Herman, T. Strohmer, High-resolution radar via compressed sensing IEEE Trans. Signal Process. 57(6), 2275-2284 (2009)

11. J.H.G. Ender, On compressive sensing applied to radar. Signal Process. 90(5), 1402-1414 (2010)

12. R. Baraniuk, P. Steeghs, in IEEE Radar Conference, in Compressive radar imaging (, Boston, MA, 2007), pp. 128-133

doi:10.1186/1687-1499-2012-257

Cite this article as: Wang and Zhang: Adaptive sampling with Bayesian compressive sensing in radar sensor networks and image. EURASIP Journal on Wireless Communications and Networking 2012 2012:257.

\section{Submit your manuscript to a SpringerOpen ${ }^{\circ}$ journal and benefit from:}

- Convenient online submission

- Rigorous peer review

- Immediate publication on acceptance

- Open access: articles freely available online

- High visibility within the field

- Retaining the copyright to your article

Submit your next manuscript at $>$ springeropen.com 